\title{
NONTRIVIAL SOLUTIONS FOR BOUNDARY-VALUE PROBLEMS OF NONLINEAR FRACTIONAL DIFFERENTIAL EQUATIONS
}

\author{
YingXin GuO
}

\begin{abstract}
In this paper, we consider the existence of nontrivial solutions for the nonlinear fractional differential equation boundary-value problem (BVP)

$$
\begin{gathered}
-\mathbf{D}_{0+}^{\alpha} u(t)=\lambda[f(t, u(t))+q(t)], \quad 0<t<1 \\
u(0)=u(1)=0,
\end{gathered}
$$

where $\lambda>0$ is a parameter, $1<\alpha \leq 2, \mathbf{D}_{0+}^{\alpha}$ is the standard RiemannLiouville differentiation, $f:[0,1] \times \mathbb{R} \rightarrow \mathbb{R}$ is continuous, and $q(t)$ : $(0,1) \rightarrow[0,+\infty)$ is Lebesgue integrable. We obtain serval sufficient conditions of the existence and uniqueness of nontrivial solution of BVP when $\lambda$ in some interval. Our approach is based on Leray-Schauder nonlinear alternative. Particularly, we do not use the nonnegative assumption and monotonicity which was essential for the technique used in almost all existed literature on $f$.
\end{abstract}

\section{Introduction}

Fractional differential equations have been of great interest recently. It is caused both by the intensive development of the theory of fractional calculus itself and by the applications of such constructions in various sciences such as physics, mechanics, chemistry, engineering, etc. For details, see $[1,2,4,6,7$, $8,9]$ and references therein.

Recently, there are some papers deal with the existence and multiplicity of solutions (or positive solutions) of nonlinear initial fractional differential equations by the use of techniques of nonlinear analysis (fixed-point theorems, Leray-Shauder theory, etc.), see [2, 8, 9]. However, as far as we know, there has few papers which deal with the boundary-value problem for nonlinear fractional

Received October 1, 2008.

2000 Mathematics Subject Classification. 34B15, 34B05, 26 A33.

Key words and phrases. standard Riemann-Liouville differentiation, fractional differential equation, boundary-value problem, nontrivial solution, Leray-Schauder nonlinear alternative.

The author was supported financially by the NNSF of China (10801088) and the NSF of Shandong Province of China (Q2007A02). 
differential equation. Here, we consider the existence of nontrivial solutions for the nonlinear fractional differential equation boundary-value problem (BVP)

$$
\begin{gathered}
-\mathbf{D}_{0+}^{\alpha} u(t)=\lambda[f(t, u(t))+q(t)], \quad 0<t<1 \\
u(0)=u(1)=0,
\end{gathered}
$$

where $\lambda>0$ is a parameter, $1<\alpha \leq 2$ is a real number, $\mathbf{D}_{0+}^{\alpha}$ is the standard Riemann-Liouville differentiation, $f:[0,1] \times \mathbb{R} \rightarrow \mathbb{R}$ is continuous, and $q(t)$ : $(0,1) \rightarrow[0,+\infty)$ is Lebesgue integrable.

In [2], the authors consider the existence and multiplicity of positive solutions of nonlinear fractional differential equation boundary-value problem

$$
\begin{gathered}
D_{0+}^{\alpha} u(t)+f(t, u(t))=0, \quad 0<t<1 \\
u(0)=u(1)=0,
\end{gathered}
$$

where $1<\alpha \leq 2$ is a real number. $D_{0+}^{\alpha}$ is the standard Riemann-Liouville fractional derivative, and $f:[0,1] \times[0,+\infty) \rightarrow[0,+\infty)$ is continuous. By means of some fixed-point theorems on cone, they obtained some existence and multiplicity results of positive solutions. But, they must use the nonnegative assumption on $f$.

Motivated by the work and the reason mentioned above, in this paper, we establish serval sufficient conditions of the existence of nontrivial solutions for the above nonlinear fractional differential equations (1.1). Here, by a nontrivial solution of (1.1) we understand a function $u(t) \not \equiv 0$ which satisfies (1.1).

In this paper, without any monotone-type and nonnegative assumption, we obtain serval sufficient conditions of the existence and uniqueness of nontrivial solution of BVP when $\lambda$ in some interval. Our results are new. Particularly, we do not use the nonnegative assumption and monotonicity which was essential for the technique used in almost all existed literature on $f$.

\section{Preliminaries}

For completeness, in this section, we present here the necessary definitions from fractional calculus theory. These definitions can be found in the recent literature.

Definition 2.1 ([7, Definition 2.1]). The integral

$$
I_{0+}^{s} f(x)=\frac{1}{\Gamma(s)} \int_{0}^{x} \frac{f(t)}{(x-t)^{1-s}} d t, \quad x>0,
$$

where $s>0$, is called Riemann-Liouville fractional integral of order $s$.

Definition 2.2 ([7, pp. 36-37]). For a function $f(x)$ given in the interval $[0, \infty)$, the expression

$$
D_{0+}^{s} f(x)=\frac{1}{\Gamma(n-s)}\left(\frac{d}{d x}\right)^{n} \int_{0}^{x} \frac{f(t)}{(x-t)^{s-n+1}} d t,
$$

where $n=[s]+1,[s]$ denotes the integer part of number $s$, is called the RiemannLiouville fractional derivative of order $s$. 
Lemma 2.1 ([2, Lemma 2.3]). Let $h(t) \in C[0,1]$ be a given function and $1<\alpha \leq 2$. Then the boundary-value problem

$$
\begin{gathered}
-\mathbf{D}_{0+}^{\alpha} u(t)=h(t), \quad 0<t<1 \\
u(0)=u(1)=0
\end{gathered}
$$

has a unique solution

$$
u(t)=\int_{0}^{1} G(t, s) h(s) d s
$$

where

$$
G(t, s)= \begin{cases}\frac{[t(1-s)]^{\alpha-1}-(t-s)^{\alpha-1}}{\Gamma(\alpha)}, & s \leq t, \\ \frac{[t(1-s)]^{\alpha-1}}{\Gamma(\alpha)}, & t \leq s .\end{cases}
$$

Here $G(t, s)$ is called the Green's function of boundary-value problem (2.1).

Lemma 2.2. Let $h(t) \in C[0,1]$ be a given function. Then function $G(t, s)$ defined by (2.3) has the following properties:

$(\mathrm{R} 1) G(t, s) \in C([0,1] \times[0,1))$, and $G(t, s) \geq 0$ for $\forall t, s \in[0,1]$;

(R2) $G(t, s) \leq \frac{[s(1-s)]^{\alpha-1}}{\Gamma(\alpha)}$ for $\forall t, s \in[0,1]$.

Proof. The proof of this lemma is easy, and we omit it.

Lemma $2.3([3,5])$. Let $X$ be a real Banach space, $\Omega$ be a bounded open subset of $X, 0 \in \Omega, T: \bar{\Omega} \rightarrow X$ is a completely continuous operator. Then, either there exist $x \in \partial \Omega, \mu>1$ such that $T(x)=\mu x$, or there exists a fixed point $x^{*} \in \bar{\Omega}$.

\section{Main results}

We put $X=C[0,1]$ endowed with the ordering $x \leq y$ if $x(t) \leq y(t)$ for all $t \in[0,1]$, and $\|u\|=\max _{t \in[0,1]}|u(t)|$ is defined as usual by maximum norm. Clearly, it follows that $(X,\|\cdot\|)$ is a Banach space.

We also need the following conditions and assumptions for our main results:

(A) $\int_{0}^{1}[t(1-t)]^{\alpha-1} q(t) d t<+\infty$.

(B) $f(t, 0) \not \equiv 0$, and there exist nonnegative functions $p, r \in L[0,1]$ such that

$$
|f(t, u)| \leq p(t)|u|+r(t), \text { a.e. }(t, u) \in[0,1] \times \mathbb{R},
$$

and there exists $t_{0} \in[0,1]$ such that $p\left(t_{0}\right) \neq 0$.

Lemma 3.1. Assume that (A) holds. Let $T: X \rightarrow X$ be the operator defined by

$$
T u(t):=\lambda \int_{0}^{1} G(t, s)[f(s, u(s))+q(s)] d s .
$$

Then $T: X \rightarrow X$ is completely continuous. 
Proof. The operator $T: X \rightarrow X$ is continuous in view of nonnegativeness and continuity of $\mathrm{G}(\mathrm{t}, \mathrm{s})$ and continuity of $[f(t, u)+q(t)]$.

Let $\Omega \in X$ be bounded, i.e., there exists a positive constant $M>0$ such that $\|u\|<M$ for all $u \in \Omega$. Let $L=\max _{0 \leq t \leq 1,0 \leq u \leq M}|f(t, u)|$. Then, for $u \in \Omega$, we have

$$
\begin{aligned}
T u(t) & =\lambda \int_{0}^{1} G(t, s)[f(s, u(s))+q(s)] d s \\
& \leq \lambda L \int_{0}^{1}[s(1-s)]^{\alpha-1} d s+\lambda \int_{0}^{1}[s(1-s)]^{\alpha-1} q(s) d s \\
& <+\infty .
\end{aligned}
$$

Hence, $T(\Omega)$ is bounded.

On the other hand, given any $\varepsilon>0$, setting

$$
\delta=\left\{\left[\lambda \frac{L}{\alpha}+\lambda \int_{0}^{1}(1-s)^{\alpha-1} q(s) d s\right]^{-1} \varepsilon\right\}^{\frac{1}{\alpha-1}},
$$

then, for each $u \in \Omega, t_{1}, t_{2} \in[0,1], t_{1}<t_{2}$, and $t_{2}-t_{1}<\delta$, one has

$$
\begin{aligned}
& \left|T u\left(t_{2}\right)-T u\left(t_{1}\right)\right| \\
\leq & \lambda \int_{0}^{1}\left|G\left(t_{2}, s\right)-G\left(t_{1}, s\right)\right||f(s, u(s))+q(s)| d s \\
\leq & \lambda \int_{0}^{1}\left|G\left(t_{2}, s\right)-G\left(t_{1}, s\right)\right|(L+q(s)) d s \\
\leq & \left(\lambda L \int_{0}^{1}(1-s)^{\alpha-1} d s+\lambda \int_{0}^{1}(1-s)^{\alpha-1} q(s) d s\right)\left(t_{2}^{\alpha-1}-t_{1}^{\alpha-1}\right) \\
\leq & \left(\lambda \frac{L}{\alpha}+\lambda \int_{0}^{1}(1-s)^{\alpha-1} q(s) d s\right)\left(t_{2}-t_{1}\right)^{\alpha-1} \\
\leq & \varepsilon
\end{aligned}
$$

That is to say, $T(\Omega)$ is equicontinuity.

By the Arzela-Ascoli theorem, we have $T: X \rightarrow X$ is completely continuous. The proof is complete.

Theorem 3.1. Suppose that (A) and (B) hold. Then there exists a constant $\lambda^{*}>0$ such that for any $0<\lambda \leq \lambda^{*}$, the $\mathrm{BVP}(1.1)$ has at least one nontrivial solution $u^{*} \in C[0,1]$.

Proof. By Lemma 2.1, problem (1.1) has a solution $u=u(t)$ if and only if $u$ solves the operator equation

$$
u(t)=T u(t):=\lambda \int_{0}^{1} G(t, s)[f(s, u(s))+q(s)] d s
$$

in $X$. So we need to seek a fixed point of $T$ in $X$. By Lemma 3.1, the operator $T: X \rightarrow X$ is a completely continuous operator. 
Since $|f(t, 0)| \leq r(t)$, a.e. $t \in[0,1]$, we know $\int_{0}^{1}[r(t)+q(t)] d t>0$. From $p\left(t_{0}\right) \neq 0$, we easily obtain $\int_{0}^{1} p(s) d s>0$. Let

$$
m=\frac{\int_{0}^{1}[s(1-s)]^{\alpha-1}[r(s)+q(s)] d s}{\int_{0}^{1}[s(1-s)]^{\alpha-1} p(s) d s}, \Omega=\{u \in C[0,1]:\|u\|<m\} .
$$

Suppose $u \in \partial \Omega, \mu>1$ such that $T u=\mu u$. Then

$$
\mu m=\mu\|u\|=\|T u\| .
$$

Since

$$
\begin{aligned}
& \|T u\| \\
= & \max _{0 \leq t \leq 1}|T u(t)| \\
\leq & \max _{0 \leq t \leq 1} \lambda \int_{0}^{1} G(t, s)|f(s, u(s))+q(s)| d s \\
\leq & \frac{\lambda}{\Gamma(\alpha)} \int_{0}^{1}[s(1-s)]^{\alpha-1}|f(s, u(s))+q(s)| d s \\
\leq & \frac{\lambda}{\Gamma(\alpha)} \int_{0}^{1}[s(1-s)]^{\alpha-1}[p(s)|u(s)|+r(s)+q(s)] d s \\
\leq & \frac{\lambda}{\Gamma(\alpha)} \int_{0}^{1}[s(1-s)]^{\alpha-1} p(s) d s|| u \|+\frac{\lambda}{\Gamma(\alpha)} \int_{0}^{1}[s(1-s)]^{\alpha-1}[r(s)+q(s)] d s .
\end{aligned}
$$

Choose $\lambda^{*}=\frac{\Gamma(\alpha)}{2 \int_{0}^{1}[s(1-s)]^{\alpha-1} p(s) d s}$. Then when $0<\lambda \leq \lambda^{*}$, we have

$$
\mu\|u\| \leq \frac{1}{2}\|u\|+\frac{\int_{0}^{1}[s(1-s)]^{\alpha-1}[r(s)+q(s)] d s}{2 \int_{0}^{1}[s(1-s)]^{\alpha-1} p(s) d s} .
$$

Consequently,

$$
\mu \leq \frac{1}{2}+\frac{\int_{0}^{1}[s(1-s)]^{\alpha-1}[r(s)+q(s)] d s}{2 m \int_{0}^{1}[s(1-s)]^{\alpha-1} p(s) d s}=1 .
$$

This contradicts $\mu>1$, by Lemma 2.3, $T$ has a fixed point $u^{*} \in \bar{\Omega}$, since $f(t, 0) \not \equiv 0$, then when $0<\lambda \leq \lambda^{*}$, the $\operatorname{BVP}(1,1)$ has a nontrivial solution $u^{*} \in C[0,1]$. This completes the proof.

If we use the following stronger condition than (B), we can obtain the following corollary.

Corollary 3.1. Suppose that $f(t, 0) \not \equiv 0$, and there exist nonnegative functions $p \in L[0,1]$ such that

$$
\left|f\left(t, u_{1}\right)-f\left(t, u_{2}\right)\right| \leq p(t)\left|u_{1}-u_{2}\right| \text {, a.e. }\left(t, u_{i}\right) \in[0,1] \times \mathbb{R}(i=1,2),
$$

and there exists $t_{0} \in[0,1]$ such that $p\left(t_{0}\right) \neq 0$. Then there exists a constant $\lambda^{*}>0$ such that for any $0<\lambda \leq \lambda^{*}$, the $\mathrm{BVP}(1.1)$ has an unique nontrivial solution $u^{*} \in C[0,1]$. 
Proof. In fact, if $u_{2}=0$, then we have $|f(t, u)| \leq p(t)|u|+f(t, 0)$, a.e. $(t, u) \in$ $[0,1] \times \mathbb{R}$. From Theorem 3.1, we know the $\operatorname{BVP}(1.1)$ has a nontrivial solution $u^{*} \in C[0,1]$.

But in this case, we prefer to concentrate uniqueness of nontrivial solution for the $\operatorname{BVP}(1.1)$. Let $T$ be given in Theorem 3.1, we shall show that $T$ is a contraction. In fact

$$
\begin{aligned}
\left\|T u_{1}-T u_{2}\right\| & =\max _{0 \leq t \leq 1} \lambda \mid \int_{0}^{1} G(t, s)\left(f\left(t, u_{1}(s)\right)-f\left(t, u_{2}(s)\right) d s \mid\right. \\
& \leq \frac{\lambda}{\Gamma(\alpha)} \int_{0}^{1}[s(1-s)]^{\alpha-1}\left|f\left(t, u_{1}(s)\right)-f\left(t, u_{2}(s)\right)\right| d s \\
& \leq \frac{\lambda}{\Gamma(\alpha)} \int_{0}^{1}[s(1-s)]^{\alpha-1}\left[p(s)\left|u_{1}(s)-u_{2}(s)\right|\right] d s \\
& \leq \frac{\lambda}{\Gamma(\alpha)} \int_{0}^{1}[s(1-s)]^{\alpha-1} p(s) d s\left\|u_{1}-u_{2}\right\| .
\end{aligned}
$$

If we choose $\lambda^{*}=\frac{\Gamma(\alpha)}{2 \int_{0}^{1}[s(1-s)]^{\alpha-1} p(s) d s}$, then, when $0<\lambda \leq \lambda^{*}$, we have

$$
\left\|T u_{1}-T u_{2}\right\| \leq \frac{1}{2}\left\|u_{1}-u_{2}\right\| .
$$

So $T$ is indeed a contraction. Finally, we use the Banach fixed point theorem to deduce the existence of an unique solution to the $\operatorname{BVP}(1.1)$.

Theorem 3.2. Suppose that $f(t, 0) \not \equiv 0$, and

$$
0 \leq M=\limsup _{|u| \rightarrow+\infty} \max _{0 \leq t \leq 1} \frac{|f(t, u)|}{|u|}<+\infty .
$$

Then there exists a constant $\lambda^{*}>0$ such that for any $0<\lambda \leq \lambda^{*}$, the $\operatorname{BVP}(1.1)$ has at least one nontrivial solution $u^{*} \in C[0,1]$.

Proof. Let $\varepsilon>0$ such that $M+1-\varepsilon>0$. By (3.1), there exists $H>0$ such that

$$
|f(t, u)| \leq(M+1-\varepsilon)|u|,|u| \geq H, 0 \leq t \leq 1 .
$$

Let $N=\max _{t \in[0,1],|u| \leq H}|f(t, u)|$. Then for any $(t, u) \in[0,1] \times \mathbb{R}$. We have

$$
|f(t, u)| \leq(M+1-\varepsilon)|u|+N .
$$

From Theorem 3.1 we know the $\operatorname{BVP}(1.1)$ has at least one nontrivial solution $u^{*} \in C[0,1]$.

\section{Examples}

Example 4.1. Consider the following second-order boundary value problem:

$$
\left\{\begin{array}{l}
-\mathbf{D}_{0+}^{1.5} y(t)=\lambda\left(\frac{y \sin t}{\sqrt{t(1-t)}}+t(1+t)\right)+\lambda t^{3}, \quad 0<t<1, \\
y(0)=y(1)=0
\end{array}\right.
$$


In this example $f(t, y(t))=\frac{y \sin t}{\sqrt{t(1-t)}}+t(1+t)$, then

and

$$
|f(t, y(t))| \leq \frac{1}{\sqrt{t(1-t)}}|y|+t(1+t)
$$

$$
\frac{\Gamma\left(\frac{3}{2}\right)}{2 \int_{0}^{1}[s(1-s)]^{\left(\frac{3}{2}-1\right)} \frac{1}{\sqrt{s(1-s)}} d s}=\frac{\Gamma\left(\frac{3}{2}\right)}{2}=\frac{\sqrt{\pi}}{4} .
$$

Choose $\lambda^{*}=\frac{\sqrt{\pi}}{4}$, then by Theorem 3.1, (4.1) has a nontrivial solution $y^{*} \in$ $C[0,1]$ for any $\lambda \in\left(0, \frac{\sqrt{\pi}}{4}\right]$.

Example 4.2. Consider the following second-order boundary value problem:

$$
\left\{\begin{array}{l}
-\mathbf{D}_{0+}^{1.5} y(t)=\frac{3 \lambda \sqrt{t(1-t)}}{4}(y-\cos y)+\lambda t e^{2 t-1}, \quad 0<t<1 \\
y(0)=y(1)=0
\end{array}\right.
$$

In this example $f(t, y(t))=\frac{3 \sqrt{t(1-t)}}{4}(y-\cos y)$, then

$$
\left|f\left(t, y_{1}(t)\right)-f\left(t, y_{2}(t)\right)\right| \leq p(t)\left|y_{1}-y_{2}\right|,
$$

where $p(t)=\frac{3 \sqrt{t(1-t)}}{2}$. By computation, we get $\lambda^{*}=2 \Gamma(1.5)=\sqrt{\pi}$. Choose $\lambda^{*}=\sqrt{\pi}$, then by Corollary $3.1,(4.2)$ has a unique nontrivial solution $y^{*} \in$ $C[0,1]$ for any $\lambda \in(0, \sqrt{\pi}]$.

\section{References}

[1] O. P. Agrawal, Formulation of Euler-Lagrange equations for fractional variational problems, J. Math. Anal. Appl. 272 (2002), no. 1, 368-379.

[2] Z. Bai and H. Lü, Positive solutions for boundary value problem of nonlinear fractional differential equation, J. Math. Anal. Appl. 311 (2005), no. 2, 495-505.

[3] K. Deimling, Nonlinear Functional Analysis, Springer-Verlag, Berlin, 1985.

[4] D. Delbosco and L. Rodino, Existence and uniqueness for a nonlinear fractional differential equation, J. Math. Anal. Appl. 204 (1996), no. 2, 609-625.

[5] B. Liu, Positive solutions of a nonlinear three-point boundary value problem, Comput. Math. Appl. 44 (2002), no. 1-2, 201-211.

[6] I. Podlubny, Fractional Differential Equations, Mathematics in Science and Engineering, 198. Academic Press, Inc., San Diego, CA, 1999.

[7] S. G. Samko, A. A. Kilbas, and O. I. Marichev, Fractional Integral and Derivatives, Gordon and Breach, Switzerland, 1993.

[8] S. Zhang, The existence of a positive solution for a nonlinear fractional differential equation, J. Math. Anal. Appl. 252 (2000), no. 2, 804-812.

[9] _ Existence of positive solution for some class of nonlinear fractional differential equations, J. Math. Anal. Appl. 278 (2003), no. 1, 136-148.

Department of Mathematics

Qufu Normal University

Qufu, Shandong 273165, P. R. China

E-mail address: yxguo312@163.com 